\title{
Zum \\ akuten fettembolischen Tode nach leichten Erschütterungen ${ }^{1}$ ). \\ Von \\ Prof. Ernst Ziemke-Kiel.
}

M. H.-Fettembolien gehören bekanntlich nicht zu den seltenen Obduktionsbefunden. Scriba fand sie in 52\% des Freiburger Obduktionsmaterials und Carrara konnte sie an 102 Leichen der verschieden. sten Herkunft, die in das Wiener Institut für gerichtliche Medizin eingeliefert worden waren, in $26,4 \%$ der Fälle feststellen. Als häufigste Ursache kommen Knochenbrüche in Betracht - Carrara fand sie in $76 \%$ seines Materials - nächstdem schwere Quetschungen von fettreichen Weichteilen. Aber auch leichtere allgemeine Erschütterungen des Körpers können unter Umständen zu erheblichen Fettembolien in den Lungen und anderen Organen Anlaß geben. Dies wird ohne Zweifel durch die Untersuchungen und Beobachtungen von Ribbert, Busse, Eichhorn u. a. bewiesen.

Bei der Häufigkeit des Vorkommens von Fettembolien an Leichen erscheint eine gewisse Kritik in der Beurteilung ihres ursächlichen Zusammenhanges mit dem Eintritt eines akuten Todes sicherlich geboten. Es ist daher begreiflich, daß von mancher Seite davor gewarnt wird, die Fettembolie allein als Todesursache anzusehen, und daß man ihr solche Wirkung nur in seltenen Fällen zuerkennen will. Immerhin sind eine Reihe von Fällen veröffentlicht worden, die zu beweisen scheinen, daß hier lediglich die ausgedehnten Fettembolien in den verschiedenen Organen den tödlichen Ausgang verursacht haben.

Ein Teil dieser Beobachtungen betrifft Fälle von Fettembolien, die im Anschluß an ein Trauma mit deutlich erkennbaren örtlichen Folgen oder an Verletzungen des Unterhautfettgewebes in kurzer Zeit akut tödlich endigten. So berichtet Zwicke von eịnem 72jährigen Mann, bei dem der Tod nach einer Humerusfraktur am 4. Tage erfolgte und mikroskopisch hochgradige Fettembolie der Lungen mit Fettentartung des Herzmuskels gefunden wurde. In einem anderen Fall von Schenkelhalsbruch bei einem 27jährigen Mann mit unerwartetem Tode am folgenden Tage und ausgedehnten Fettembolien beider Lungen ist das

1) Vorgetragen auf der Versammlung der Deutschen Gesellschaft für gerichtl. und soziale Medizin in Erlangen, September 1921.

Z. f. d. ges. geriehtl. Medjzin. Bd. 1 . 
Vorliegen eines Chloroformtodes wohl nicht ganz ausgeschlossen. Hämig beschreibt weitere Fälle. In dem einen trat nach einer Tibiafraktur der Tod am 6. Tage ein; im Gehirn wurden neben zahlreichen punktförmigen Blutungen Fettembolien gefunden. In einem anderen kam es bei einer Femurfraktur nach 42 Stunden zum Tode. Die Obduktion ergab zahlreiche miliare Blutungen im Großhirn, Fettembolie des Herzens und der Lungen. Drei andere Fälle, die Hämig noch anführt, zeigten bei an sich geringfügigen Verletzungen ausgedehnte Fettembolien in Lungen und Gehirn und endigten innerhalb der nächsten 48 Stunden mit dem Tode. Eberth sah bei einem sonst völlig gesunden Menschen nach Oberschenkelzertrümmerung in den Lungen nur wenige Fettembolien und Blutungen, eine größere Menge dagegen im Gehirn. In einem zweiten von ihm mitgeteilten Falle war ein bleichsüchtiges Mädchen, bei dem nach gewaltsamer Streckung der Kniegelenke eine geringe Knochenverletzung eingetreten war, bald darauf unter Bluthusten verstorben. Trotz der Geringfügigkeit der Knochenverletzung allerdings bei sehr fettreichem Knochenmark waren die Lungencapillaren in großer Ausdehnung mit Fett vollgestopft. In einem Fall von Fromberg wurde ein Kutscher in bewußtlosem Zustande mit einem Oberschenkelbruch in die Klinik eingeliefert. Nach vorübergehender Besserung der Benommenheit trat am folgenden Tage wieder tiefe Bewußtlosigkeit, weiterhin eine Lähmung des rechten Arms und Beins und am 5. Tage unter zunehmender Temperatursteigerung bis $40^{\circ}$, beschleunigter Atmung und starken bronchitischen Geräuschen der Tod ein. Die Obduktion ergab sehr zahlreiche capillare Hirnblutungen mit ausgedehnten Fettembolien im Gehirn mehr oder minder reichliche Fettembolien in den anderen Organen und fettige Entartung des Herzmuskels.

In einer Reihe von Fällen waren dem Tode unblutige orthopädische Eingriffe vorangegangen. So bei einem 16 jährigen Mädchen Payrs, das nach Streckung einer Kniecontractur kleinen Puls und Dyspnoe bekam und nach 26 Stunden starb. Neben akutem Lungenödem wurden hochgradige Fettembolien der Lungen, Fettentartung des Herzens, der Nieren und der Leber gefunden. In einem Falle von Ahrens trat der Tod nach gewaltsamer Streckung beider Kniegelenke noch am gleichen Tage abends unter Somnolenz und Fieber von $39^{\circ}$ ein. In den ödematösen und hinten atelektatischen Lungen waren weitverbreitete Fettembolien vorhanden. Ahrens erwähnt auch den Fall eines 8jährigen Mädchens, bei dem nach gewaltsamer Streckung zahlreicher Gelenke der Tod am 3. Tage unter Dyspnoe eintrat und sich ausgedehnte Fettembolien der Lungen, des Herzmuskels und der Nieren fanden. Lympius sah schon 5 Minuten nach der gewaltsamen Streckung eines kontrahierten Kniegelenks den Tod durch vollständige t'berschwemmung der 
Lungengefäße mit flüssigem Fett eintreten, $M e c h$ in 2 anderen Fällen 4 bzw. 6 Stunden nach einem Sturz aus dem Fenster bei Oberschenkelfraktur. Colley berichtet über ein 26jähriges Mädchen, das 7 Stunden nach der gewaltsamen Gelenkstreckung komatös wurde und 14 Stunden nach der Operation unter den Erscheinungen des Lungenödems zugrunde ging. Mikroskopisch zeigten die Lungen, Gehirn und Nieren ausgedehnte Fettembolien. Zahlreiche kleine blaßgelbe Flecken an der Innenseite der Herzkammern zum Teil mit zentralem rotem Punkt erwiesen sich als stark fettig entartete Partien der Herzkusmulatur, in denen sehr zahlreiche Capillaren mit unregelmäßigen länglichen Fettropfen angefüllt waren. Im Magen, Darm und Milz waren ebenfalls größere Bezirke mit Fett gefüllt, während solches in den Lebergefäßen vermißt wurde. Besonders auffällig erschien es, daß durch die Füllung der Herzcapillaren mit Fett bereits innerhalb von 14 Stunden als Folge der schlechten Ernährung eine akute Verfettung des Herzmuskels eingetreten war. In einem Fall, über den Brodbeck berichtet, war nach mehrfacher lang. samer und vorsichtiger Streckung der Gelenke Fieber bis $38,8^{\circ}$ und mehrfach Erbrechen aufgetreten. Bei einer neuen in Chloroformnarkose vorgenommenen gewaltsamen Streckung der Knie und Füße starb der Kranke 10 Minuten nach der Operation ganz plötzlich. Die mikroskopische Untersuchung zeigte ausgedehnte capilläre Fettembolie der Lungen, sonst keinen pathologischen Befund. Auch von Gaugele werden 2 tödliche Fälle von Fettembolie nach orthopädischen Operationen mitgeteilt.

Diesen Beobachtungen stehen nun andere gegenüber, wo zwar auch als Ursache des Todes lediglich ausgedehnte Fettembolien in verschiedenen lebenswichtigen Organen gefunden wurden, aber ohne da $B$ eine wesentliche Spur vorangeganger Gewalteinwirkung vorhanden gewesen wäre.

Busse teilt folgenden Fall mit, der für die Unfallpraxis von besonderem Interesse ist. Ein ungewöhnlich fettleibiger Mann fiel eine 12 Stufen hohe Treppe herunter und arbeitete am nächsten Morgen wieder am Schreibtisch, ohne noch Folgen des Unfalls zu verspüren. Plötzlich wurde er unwohl, legte sich wieder ins Bett und starb nach 20 Minuten. Als Ursache des plötzlichen Todes ergab die Obduktion eine capilläre Fettembolie der Lungen und fettige Metamorphose des Herzmuskels. Ein zweiter Fall Busses ist dadurch bemerkenswert, daß die tödliche Fettembolie durch den Transport des Verletzten eine $25 \mathrm{~km}$ lange Strecke auf einem federlosen Kastenwagen und zum Teil recht holperigen Wege erfolgte. Ein 65jähriger Kuhhirte hatte durch den Stoß eines Bullen einen Bruch des Brustbeins und mehrerer Rippen erlitten. Obwohl er sich von dem Unfall bald wieder erholt hatte, wurde er doch auf seinen Wunsch zur besseren Ausheilung in die Greifswalder 
chirurgische Klinik überführt. Die Erschütterungen und Stöße beim Fahren verursachten ihm Schmerzen, er fragte immer ungeduldiger, ob man nicht bald am Ziele sei, wurde allmählich stiller und bald ganz still. Als man in der Klinik ankam, war er tot. Bei der Obduktion fand man sehr starke capilläre Fettembolie in allen Teilen der Lungen, auch die Capillarschlingen der Glomeruli in den Nieren enthielten reichlich Fett, im Gehirn ließ sich dagegen kein Fett nachweisen. Bei dem Fehlen eines anderen pathologischen Befundes konnte nur die Fettembolie für den Tod verantwortlich gemacht werden. Auch Kaufmann erwähnt einen Todesfall nach Transport eines Schwerverletzten auf der Eisenbahn am 4. Tage nach dem Unfall. In einer Beobachtung Ribberts konnte nur aus einer handtellergroßen Epidermisabschürfung und oberflächlichen Blutung in das Zellgewebe über dem linken Trochanter vermutet werden, daß vor dem Tode eine Quetschung stattgefunden hatte. Ein 63jähriger Mann wurde krank auf der Straße gefunden und war bald darauf gestorben. Als einzige nennenswerte Organveränderung fanden sich eitrige Herde im Nierengewebe, die von einer Nierenbecken- und Harnblasenentzündung ausgingen und für den Tod nicht in Frage kamen. Außerdem wurde aber ein hochgradiges Lungenödem und bei mikroskopischer Untersuchung eine ausgedehnte Fettembolie der Lungen und Fettanfüllung vieler Glomerulussehlingen der Nieren festgestellt. Endlich ist noch ein Fall ähnlicher Art von Eichhorn veröffentlicht. Ein 71 jähriger Arbeiter wurde infolge eines Hufschlages taumelig, fiel rücklings über die Wagendeichsel auf das Hofpflaster und schlug dabei mit dem Kopf auf die Bordkante auf. Er erholte sich bald, ging nach Hause, klagte über Schmerzen im Rücken und Kopf und wurde 3 Stunden nach dem Unfall bewuBtlos. Bei der ärztlichen Untersuchung fand sich sehr beschleunigter kleiner Puls, beschleunigte lautrasselnde Atmung und als äußeres Zeichen des Falles eine Hautabschürfung am rechten Ellbogen. Einige Stunden später verstarb der Mann unter den Erscheinungen hochgradiger Herzschwäche. Bei der Obduktion fand man neben einer stärkeren Arteriosklerose und einigen Alterserscheinungen als wesentlichen Befund eine Hypertrophie und erhebliche Erweiterung der rechten Herzkammer, Emphysem und Odem der Lungen und mikroskopisch eine hochgradige Fettembolie der feinen und feinsten Gefäße in beiden Lungen, in Herz, Leber und Nieren.

Bei einer Beobachtung Justys, wo sich bei einem 51 jährigen Manne neben Tuberkulose und frischer Lungenentzündung ausgedehnte Fettembolie in den Lungen fand, war eine greifbare Ursache für die Fettembolie überhaupt nicht vorhanden, es konnten nur kleine unbemerkt gebliebene Quetschungen des Unterhautfettgewebes, wie sie bei Eklampsie und Delirien vorkommen können, als mögliche Ursache ihrer Ent- 
stehung vermutet werden. In einem von Engel beschriebenen Falle, der ebenfalls eine tuberkulöse Lunge betraf, war die akute tödliche Fettembolie durch eine Leberruptur entstanden. Ein 44jähriger Mann glitt beim Spazierengehen aus, wurde beim Fallen aufgefangen und machte mit dem Oberkörper eine ruckweise Drehung nach rechts. Er ging ohne Stütze in sein im zweiten Stock gelegenes Zimmer, erkrankte mit Fieber, angestrengter, etwas beschleunigter Atmung, Hustenreiz, kleinem sehr beschleunigten Puls und starb unter zunehmender Dyspnoe im Verlauf des nächsten Tages. Die Obduktion stellte neben der Tuberkulose Lungenödem und capilläre Fettembolie der Lungen und einen $10 \mathrm{~cm}$ langen, stark fetthaltigen Leberriß fest. Im mikroskopischen Bilde sah man mechanisch ausgepreßtes Fett frei zwischen den Leberzellen liegen. Offenbar waren hiervon größere Mengen mit dem Zelldetritus in den Kreislauf gelangt.

Wie man sieht, sind Fälle von akut tödlichen Fettembolien nach leichten Erschütterungen bisher nur in geringer $\mathrm{Zahl}$ veröffentlicht worden. Das liegt vermutlich daran, daß in vielen Fällen von der dem Tode voraufgegangenen Erschütterung des Körpers bei der Obduktion nichts bekannt ist und es daher unterlassen wird, die Organe auf Fettembolie zu untersuchen. Vielleicht würde man die Fettembolie als Ursache akuter Todesfälle häufiger finden, wenn man sich daran gewöhnen würde, in Fällen, wo die Todesursache durch die Obduktion nicht aufgedeckt wurde, eine Untersuchung der Organe auf Fettembolie niemals zu unterlassen. Eine Beobachtung, die ich in jüngster Zeit zu machen Gelegenheit hatte, lehrt mich jedenfalls, daß solche Untersuchung oft ganz überraschende und nicht erwartete Aufschlüsse geben kann.

Der Fall, der von mir im November 1920 obduziert wurde, war folgender. Der ehemalige Kriegsgefangene Musketier H. M. war wegen Hebephrenie in die psychiatrische Abteilung des Versorgungslazaretts Kiel aufgenommen worden. Eines Tages im November wurde der 25jährige Mann vormittags am Gitterwerk des Lazarettgrundstücks auf dem Boden liegend verletzt aufgefunden. Bei Fragen versuchte er sich aufzurichten, was ihm nicht vollständig gelang. Er stöhnte und reagierte nur schlecht auf Anruf. Auf der chirurgischen Abteilung wurde festgestellt, da $\beta$ er in der Nabelgegend eine $1 \frac{1}{2} \mathrm{~cm}$ lange quergestellte Hautwunde mit ganz scharfen Rändern hatte, die anscheinend von einem Messerstich herrührte; im Krankenkittel und Hemd fanden sich entsprechende Löcher. Ein Messer konnte weder bei ihm in den Taschen, noch im Garten in der Umgebung der Auffindungsstelle gefunden werden. Der Kranke stöhnte ununterbrochen und gab auf jede Frage die gleichlautende Antwort: „Ich weiß nicht.“ Bei der sofort vorgenommenen Eröffnung der Bauchhöhle stellte es sich heraus, daß die Wunde 
gar nicht bis in die Bauchhöhle gelangt war. Unter häufigem Erbechen, starkem Hustenreiz mit Auswurf, kleinem Puls und lang. samer Abnahme der Herztätigkeit trat schließlich nach 40 Stunden der Tod ein.

Die Obduktion bestätigte, daß die in der Bauchmittellinie oberhalb des Nabels gelegene quergestellte und $11 / 2 \mathrm{~cm}$ lange Wunde der Bauch. decken nur bis nahe an das Bauchfell heranreichte, ohne die Bauchhöhle zu eröffnen. Verletzungen der Bauchorgane oder Bauchfellentzündung waren nicht vorhanden. Beide Lungen waren voluminös, schwer und succulent, von dunkelroter, hinten mehr blauroter Farbe; die hinteren Partien waren luftärmer. Uber die Schnittflächen entleerte sich reichlicher gelber bis blaßrötlicher Gewebsschaum. Auch in den Luftröhrenästen war reichliche rötliche, etwas schaumige Flüssigkeit. Das Herz war derb und fest, seine Masse $9^{1 / 2}: 10 \mathrm{~cm}$, die Herzmuskulatur braunrot, an einigen Stellen etwas gelblich und weniger durchscheinend. Die Herzklappen waren unverändert, in den Kranzschlagadern, der Aorta und den übrigen Körpergefäßen nirgends atheromatöse Veränderungen. Die Milz nicht vergrößert, ziemlich weich, graurot. Die Nieren mit reichlicher Fettkapsel und glatter Oberfläche, die Rinde etwas weniger durchscheinend, hellbräunlich, die Markstrahlen breit, die Markkegel etwas dunkler. Die Leber hellbräunlich, nicht vergrößert, der vordere Rand stumpf, die Leberzeichnung deutlich. Das Gehirn feucht, auf der Schnittfläche eine größere Zahl Blutpunkte, sonst keine Veränderungen. $\mathrm{Zu}$ beiden Seiten der Lendenwirbelsäule im Unterhautzellgewebe bis in die Muskelschichten der Gesäßbacken hinein etwas ausgedehntere Blutunterlaufungen. Das Bauchfett ziemlich stark, durchschnittlich $1 \frac{1}{2} \mathrm{~cm}$ dick.

Dieser Obduktionsbefund ließ die Todesursache zunächst vollkommen unklar. Erst als die Lungen und dann auch die übrigen Organe auf Fettembolien untersucht wurden, war eine Aufklärung möglich. Dabei fanden sich in den kleinsten Arterien und den Capillaren der Lungen ausgedehnte und vielfach zusammenhängende Fettembolien und zwar an Schnitten, die aus den verschiedensten Lungenpartien entnommen waren. An manchen Stellen hatte man den Eindruck, als ob die Gefäße künstlich injiziert worden seien. Im Herzmuskel waren ebenfalls, wenn auch in erheblich geringerem Grade, Fettverstopfungen kleiner Arterien und Capillaren vorhanden, außerdem eine herdweise fettige Metamorphose, die bei Sudanfärbung außerordentlich schön hervortrat und auf die nächste Umgebung der embolisierten Gebiete beschränkt war. In den Nieren waren nur an einzelnen Glomerulusschlingen Fettemboli zu sehen; in ihrer Nähe zeigten die Zellen der gewundenen Harnkanälchen reichliche Fettkörnung. Am spärlichsten waren die Fettverstopfungen in den Capillaren des Gehirns. An einzelnen Stellen sah man 
mikroskopisch kleinste Blutungen von runder Form, in deren Mitte sich der durch Sudan rotgefärbte Fettpfropf deutlich abhob.

Der Fall ist wohl kaum anders zu erklären, als daß der Kranke, der gegen seinen Willen auf der psychiatrischen Abteilung des Versorgungslazaretts zurückgehalten wurde, einen Fluchtversuch unternommen hatte, sich beim Uberklettern an einer Zacke der Einfriedigung am Bauche verletzt und dabei rücklings zu Boden gefallen war. Dabei war es offenbar zu einer allgemeinen Erschütterung des Körpers gekommen, die zum Ubergang von frei gewordenem Fett in die Blutbahn und so zu einer massenhaften Verstopfung der Lungencapillaren mit Fett geführt hatte. Da das kräftige Herz des körperlich völlig gesunden Mannes zunächst noch imstande war, einen Teil des Fettes durch die Lungen hindurch in den großen Kreislauf zu pressen, konnte dieses verschleppte Fett auch in die Capillaren des Gehirns, der Leber und der Nieren gelangen und hier Fettverstopfungen hervorrufen. Ein anderer Teil des Fettes war durch die Kranzschlagadern in den Herzmuskel gelangt und hatte hier zu einer Embolisierung mit Fett geführt. Sehr bemerkenswert und in dieser Beziehung durchaus mit dem von Colley veröffentlichten Fall vergleichbar war nun die Tatsache, daß die Herzmuskelzellen in der Umgebung der embolisierten Bezirke eine ausgedehnte fettige Metamophose erkennen ließen. In ganz ähnlicher Weise sah man auch an den Nieren in der Umgebung der embolisierten Glomerulusschlingen die angrenzenden Epithelzellen der gewundenen Harnkanälchen fettig entartet. Es steht wohl außer Frage, daß diese Fettentartung des Herz- und Nierenparenchyms durch Ernährungsstörungen hervorgerufen wurde, die durch die Fettverstopfung der diese Bezirke versorgenden Blutgefäße veranlaßt wurden. So kommt man zu der interessanten Feststellung, da $\beta$ die verhältnismäßig kurze Zeit von 40 Stunden, die bis zum Eintritt des Todes verging, genügte, um so schwere Gewebsschädigungen zu bewirken. Der Einwand, daß die Fettentartung des Herzens und der Nieren Krankheitszustände gewesen sind, die schon vorher bestanden haben, wird dadurch widerlegt, daß die inneren Organe des 25jährigen kräftigen jungen Menschen im übrigen vollständig gesund waren und da $B$ die Herde der fettig entarteten Zellen immer nur da vorkamen, wo Fettembolien zu sehen waren, mithin an die Bezirke der embolisierten Gefäße gebunden waren.

Dieser Fall ist somit wiederum ein Beweis dafür, wie wichtig es ist, bei Obduktionen an das mögliche Vorkommen von Fettembolien und ihre Bedeutung für die Feststellung der Todesursache zu denken. Hätten mich nicht die Kontusionsblutungen in der Gegend der Lendenwirbelsäule auf den Gedanken gebracht, in den einzelnen Organen nach Fettembolien zu fahnden, so wäre die Deutung des Falles höchstwahrscheinlich dunkel und die Todesursache unklar geblieben. 
Der Fall ist aber noch in anderer Richtung von Interesse, insofern nämlich, als er geeignet zu sein scheint, die Richtigkeit der Theorie zu unterstützen, die Ribbert über das Zustandekommen der Fettembolien aufgestellt hat.

Ribbert ist auf Grund von Tierversuchen und eigener Beobachtungen zu der Uberzeugung gekommen, daß die Knochenbrüche und Zertrümmerungen von Fettgewebe durch Quetschung für die Herkunft des Fettes bei den Fettembolien ziemlich bedeutungslos sind. Das verschleppte Fett stammt nach seiner Ansicht vielmehr aus dem gesamten Knochensystem und gelangt aus dem Knochenmark in die Blutbahn, wo es durch mehr oder weniger heftige Erschütterungen des Körpers von den sehr zarten Knochenmarksgefäßen aufgenommen wird, von denen es zweifelhaft ist, ob sie überhaupt eine Wandung besitzen. Nur so sei es erklärlich, daß nach ganz geringfügigen Knochenbrüchen und nach leichten Erschütterungen ohne jede Quetschung und Verletzung sehr erhebliche Fettembolien beobachtet werden. Ribbert ist also der Meinung, daß die Erschütterung einzelner oder aller Knochen, wie sie ja notwendig mit jedem Knochenbruch verbunden ist, die wesentliche Ursache für den Ubergang des Fettes in die Venen bildet, da $B$ das verschleppte Fett somit seiner Hauptmenge nach aus dem von dem Trauma nicht direkt getroffenen Knochenmark stammt. Und in der Tat ist es nicht recht verständlich, wie das zerquetschte Unterhautfettgewebe einer Verletzung oder das zertrümmerte Knochenmark an der Knochenbruchstelle, die man gewöhnlich für die Fettembolien verantwortlich macht, den Ausgangspunkt einer Fettembolie bilden soll und wie hier größere Mengen Fett in den Kreislauf eindringen sollen, wenn man bedenkt, daß die verletzten Gefäße ihr Blut in die Umgebung austreten lassen, durch Thromben leicht verschlossen werden und daher nicht gleichzeitig Fett aufnehmen können. Das ausgetretene Blut übt auch einen Druck auf die Umgebung aus und komprimiert einen Teil der verletzten Gefäße, die dadurch ganz oder teilweise verschlossen werden, im günstigsten Fall also nur einen geringen Bruchteil des bei der Fettembolie vorgefundenen Fettes aufnehmen können. Die Tierversuche bestätigen die Auffassung Ribberts. Fettembolien traten bei den Tierversuchen nicht ein, sobald er jede Erschütterung des Tieres vermied und die Knochen vorsichtig durch einen Schraubstock zerbrach. Andererseits konnte der Fall der Tiere von Tischhöhe zur Erde erhebliche Fettembolien in den Lungen hervorrufen, auch in Fällen, in denen die Knochen nicht verletzt waren.

Auch im vorliegenden Fall ist die Entstehung der Fettembolien wohl kaum anders zu erklären, als in der von Ribbert angegebenen Weise. Es fehlte jede Spur einer Knochenverletzung oder nennenswerten Quetschung des Unterhautfettgewebes. Die Kontusionsblutung in der 
Gegend der Lendenwirbelsäule hat schwerlich zu einer stärkeren Zerstörung von Fettgewebe Anlaß gegeben, da sie zu geringfügig war. Andererseits weist der Sitz der Kontusionsblutung in der Nähe der Lendenwirbelsäule gerade darauf hin, daß das markreiche Knochengewebe der Wirbelkörper der Gewalteinwirkung besonders ausgesetzt war und macht es verständlich, daß reichliche Mengen Fett durch die leicht verletzlichen und klaffend erhaltenen Markgefäßchen in die Blutbahn aufgenommen werden konnten.

Wie ist nun der Eintritt des Todes durch die Fettembolien zu erklären? In der Hauptsache wird man bei dem fettembolischen Tod mit drei verschiedenen Möglichkeiten zu rechnen haben, je nachdem sich die Hauptmenge des Fettes in den Lungen, im Gehirn oder im Herzen aufgespeichert hat. Herz, Lungen und Gehirn sind die drei lebenswichtigsten Organe; Herz und Gehirn sind bekanntlich außerordentlich empfindlich gegen Störungen in ihrer Ernährung, etwas weniger vielleicht die Lungen. Jedes von ihnen kann zweifellos allein durch Fettausstopfung einer größeren Zahl seiner Gefäße gezwungen werden, seine Funktion einzustellen. In den Lungen kommt wohl nur eine beträchtliche Ausdehnung der Fettembolien als eigentliche Todesursache in Betracht. $O b$ es richtig ist, wie Flatten angibt, daß eine Fettmenge, die nur ein Fủnftel des Gesichtsfelds im Mikroskop einnimmt, nicht ausreicht, um hier als Todesursache zu gelten, bleibe dahingestellt. Es wird m. E. nehr darauf ankommen, ob sich die Fettverstopfung über alle Teile der Lungen gleichmäßig verteilt und so die Funktion der respirierenden Lungenoberfläche in ihrer Gesamtheit behindert. Auch Ribbert ist der Meinung, daß ausgedehnte Fettembolien der Lungen allein den Tod herbeiführen können. Fettembolien im Gehirn und verlängerten Mark können bei der Empfindlichkeit dieser Organe für Zirkulationsstörungen sicherlich schon in geringeren Mengen einen tödlichen Ausgang zur Folge haben: Beim Herzen spielt wohl die in Anschluß an die Fettembolien sekundär auftretende fettige Entartung der Herzmuskulatur bei dem Eintritt des Todes ein entscheidende Rolle. Graham kam auf Grund von Tierversuchen zu dem Ergebnis, daß die Fettembolie in zweifacher Weise als Ursache des Todes in Betracht kommt: entweder durch Verstopfung einer großen Anzahl von Lungengefäßen in Verbindung mit der Blockierung einer kleineren Zahl von herzversorgenden Schlagadern oder durch weitverbreitete Verstopfung der feinsten Schlagadern des großen Kreislaufes. Im ersten Fall tritt der Tod an Asphyxie sehr bald nach dem Trauma ein; im zweiten Fall vergehen 3-8 Tage, ehe Herz- und Gehirnveränderungen den Tod veranlassen. Neben den Herzveränderungen sah er auch oft fettige Entartung des 'Zwerchfells. Zweifellos sind auch die individuellen Verhältnisse des Verstorbenen von Bedeutung. So läßt z. B. im vorliegenden 
Falle der allgemeine Fettreichtum - das Bauchfett war $11 / 2 \mathrm{~cm}$ dick vermuten, da $\beta$ auch das Knochenmark fettreich und so eine besondere Disposition für die Entstehung der Fettembolien gegeben war. In manchen Fällen wird man nicht die Schädigung eines einzelnen Organs allein, sondern die Gesamtheit der Fettverschleppungen in den verschiedenen Organen für den Tod verantwortlich machen müssen. Klinisch standen im vorliegenden Falle Lungen- und Herzsymptome im Vordergrund der Erscheinungen - starker Hustenreiz mit Auswurf und kleiner stark beschleunigter Puls. Wie das häufige bis zum Tode andauernde Erbrechen zu erklären ist, läßt sich schwer sagen. Es als cerebrales Symptom zu deuten, geht bei der Geringiügigkeit der Fettembolien im Gehirn und dem Fehlen von Bewußtseinsstörungen wohl nicht an; möglich, da $B$ auch in der Magenschleimhaut, die mikroskopisch nicht untersucht wurde, kapilläre Fettembolien vorhanden waren und eine Schleimhautreizung bewirkt haben. Brodbeck sah in seinem Falle ebenfalls nach wiederholten vorsichtigen Streckungen der Gelenke mehrfach Erbrechen auftreten. Mikroskopisch fanden sich nur in den Lungen ausgedehnte Fettembolien; das Gehirn war frei; die Magenschleimhaut wurde nicht untersucht. Trotz der nicht unbeträchtlichen Ausbreitung der Fettembolien in den Lungen trat der Tod in meinem Falle doch erst nach 40 Stunden unter den Zeichen allmählich zunehmender Herzschwäche ein. In letzter Linie sind für seinen Eintritt also die Veränderungen am Herzen verantwortlich $\mathrm{zu}$ machen.

Im anatomischen Befunde war nichts Charakteristisches, was das Bestehen so zahlreicher Fettembolien in den verschiedenen Organen hätte vermuten lassen. Nur das sehr starke Lungenödem in Verbindung mit der Kontusion am Rücken konnte vielleicht den Gedanken nahe legen, daß die Ursache des Zirkulationshindernisses in einer Fettverstopfung der Lungengefäße zu suchen war.

Der mitgeteilte Fall zeigt uns, wie nützlich es ist, sich bei dem Suchen nach der Todesursache auch des akuten Todes durch Fettembolie zu erinnern. Die Annahme liegt nahe, daßß mancher ungeklärte Todesfall, der mit der nichtssagenden Diagnose ,Schock, Herzschlag, Lungenschlag" abgetan wird, befriedigende Aufklärung erfahren würde, wenn die Möglichkeit einer tödlichen Fettembolie in Erwägung gezogen würde.

\section{Literatur.}

Scriba, Untersuchungen über Fettembolie, Dtsch. Zeitschr. f. Chirurg. 12. 1879. - Carrara, Fettembolie der Lungen in ihren Beziehungen zur gerichtl. Medizin. Friedreichs Bl. f. gerichtl. Med. 1898. - Ribbert, Fettembolie. Korrespondenzbl. f. Schweiz. Ärzte 1894 u. 1900; Dtsch. med. Wochenschr. 1900; Monatsschr. f. Unfallheilk. u. Invalidenw. 1900. - Busse, Fettembolie. Monatsschr. 
f. Unfallheilk. u. Invalidenw. 1901; Ärztl. Sachverst.-Zeit. 1901. - Eichhorn, Fettembolie. Inaug.-Diss. Leipzig 1907. - Zwicke, Zwei Todesfälle durch Fettembolie der Lungen. Dtsch. med. Wochenschr. 1883. - Hämig, Fettembolie des Gehirns. Bruns Beitr. z. klin. Chirurg. 2r. 1900. - Eberth, Fettembolie. Fortschr. d. Med. 16, Nr. 7. - Payr, Tödliche Fettembolie nach Streckung von Contrakturen. Münch. med. Wochenschr. 1898; Monatsschr. f. klin. Chirurg. 59, Heft 4. - Ahrens, Tödliche Fettembolie nach gewaltsamer Streckung beider Kniegelenke. Bruns Beitr. z. klin. Chirurg. 14. 1895. - Lympius, Tödliche Fettembolie bei Brisement foreé, Jahrb. d. Hamb. Staatskrankenanst. 4. - Mech, Tödliche Fettembolie nach Frakturen. Bruns Beitr. z. klin. Chirurg. 8. 1892. - Colley, Fettembolie nach gewaltsamer Gelenkbeugung. Dtsch. Zeitschr. f. Chirurg. 36. 1893. - Brodbeck, Fettembolie. Inaug.-Diss. Greifswald 1901. — Gaugele, Fettembolie nach orthopädischen Operationen. Zeitschr. f. orthop. Chirurg. 27. - Kaufmann, Handbuch der Unfallmedizin 1907. - Justy, Zur Kenntnis der hyalinen Capillarthrombose in Lungen und Nieren. Inaug.-Diss. Augsburg 1894. - Engel, Fettembolie nach Leberruptur. Münch. med. Wochenschr. 1901. - Flatten, Fettembolie. Zeitschr. f. Medizinalbeamte 1898, S. 741. - Graham, Fettembolie als Todesursache. Journ. of med. research. 1908. - Fromberg, Fettembolie des großen Blutkreislaufs und ihre Ursachen. Mitt. a. d. Grenzgeb. d. Med. u. Chirurg. 35. 1913. 